\title{
The Effect of Maternal Mathematical Activities on the Fetal Brain
}

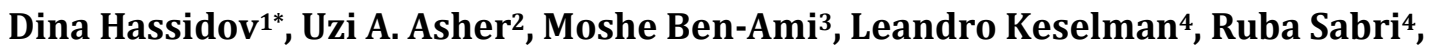 \\ Sami Haddad ${ }^{3}$ \\ ${ }^{1}$ Talpiot College of Education, Holon, Israel \\ ${ }^{2}$ Clalit Health Services, Tel Aviv, Israel \\ ${ }^{3}$ The Baruch Padeh Medical Center, Faculty of Medicine in the Galilee, Bar Ilan University, Ramat Gan, Israel \\ ${ }^{4}$ The Baruch Padeh Medical Center, Tiberias, Israel \\ Email: ^hasidov@netvision.net.il
}

How to cite this paper: Hassidov, D., Asher, U.A., Ben-Ami, M., Keselman, L., Sabri, R. and Haddad, S. (2018) The Effect of Maternal Mathematical Activities on the Fetal Brain. Open Journal of Obstetrics and Gynecology, 8, 826-835.

https://doi.org/10.4236/ojog.2018.810086

Received: July 21, 2018

Accepted: August 20, 2018

Published: August 23, 2018

Copyright $\odot 2018$ by authors and Scientific Research Publishing Inc.

This work is licensed under the Creative

Commons Attribution International

License (CC BY 4.0).

http://creativecommons.org/licenses/by/4.0/

\begin{abstract}
Background: In recent years, there has been growing interest in the effect of maternal exposure to physiological, environmental, and also psychological factors during gestation on child development. Several independent studies link maternal stress during pregnancy to emotional and behavioral problems in the child. Objectives: This study aimed to observe the effect of maternal cognitive activity on fetal brain blood flow to determine whether systematic maternal mathematical activity during pregnancy might influence child brain development. Method: Thirty-five women in the 28th to 40th week of pregnancy engaged in mathematical activities. Fetal middle cerebral artery (MCA), pulsatility index (PI) and peak systolic velocity (PSV) were monitored before, during, and after the activity. Results: Brain activity and blood flow were shown to be intimately linked. We observed a significant decrease in fetal brain MCA resistance, as evidenced by decreased MCA PI, towards the end of the mathematical activity. This may result in increased blood flow in the arteries supplying most brain regions and, possibly, increased brain activity. Conclusions: A correlation between the mother's engagement in mathematical activities and fetal brain blood flow may lead to enhancement of the fetus's brain function and a cognitive advantage for the child.
\end{abstract}

\section{Keywords}

Pregnancy, Fetal Brain, Cognitive Enhancement, Mathematical Activity, Maternal-Fetal Relationship

\section{Introduction}

While there are no direct neural connections between mother and fetus, mater- 
nal experiences are known to generate a cascade of physiological and neurochemical consequences that may directly or indirectly alter the intrauterine milieu and generate fetal response [1]. For example, studies designed to generate alarm in pregnant women [2], administration of the Stroop Color and Word test (a challenging cognitive-perceptual task) [3], or exposing women at 32 weeks of gestation to a stress-inducing documentary film [4] while evaluating fetal responsiveness to the induced maternal stress [5] [6] showed brief (four-minute) fetal responses of increased heart-rate variability and suppressed motor activity [4]. Conversely, decreased fetal heart rate and increased fetal heart rate variability were observed when women at 32 weeks of gestation underwent a relaxation procedure to reduce psychological and physiological tension. Although the fetal response may be attributed to maternal recumbency and not maternal relaxation, the clear suppression in fetal motor activity during manipulation recovered after the relaxation protocol concluded [7]. These studies demonstrate that maternal psychological changes affect fetal physiology.

Wolford et al. [8] recently investigated the relation between maternal depression during pregnancy and ADHD symptoms in their children at 3.5 years and found that depression symptoms during pregnancy predict increased maternal-reported ADHD symptoms in young children.

Animal studies have shown that exposure of fetuses to cortisol results in irreversible changes in fetal brain structure and function. The development of the hypothalamic-pituitary-adrenal axis, limbic system, and prefrontal cortex are all likely affected by antenatal maternal stress and anxiety [9].

The mechanism by which maternal manipulation (e.g. induced stress or anxiety) affects fetal physiology is relatively unclear. Hypotheses include a hormonal-based mechanism (specifically the hypothalamic-pituitary-adrenal axis, especially in cases of maternal stress or anxiety). Another suggests fetal alerting to sensory-based alterations in the intrauterine milieu: fetal heart rate responses have been observed within seconds of maternal manipulations that impinge on the intrauterine environment, including maternal postural changes [10] and auditory stimuli [11] (including sounds generated by maternal vasculature and the digestive tract, which are prominent in the uterine auditory environment [12]). Thus, it is possible that the initiation, if not the maintenance, of the fetal response to some maternal manipulations may be mediated by fetal perceptual detection of changes in the intrauterine milieu.

\subsection{Epigenetics and the Fetal Programing Hypothesis}

Recent major discoveries in the fields of epigenetics and fetal programing have indicated that stimulation or exposure to factors during a critical time window induces permanent changes in the structure and function of vital fetal organs, leading, perhaps, to prolonged or permanent changes in the child's health and behavior. For example, maternal stress or anxiety during pregnancy may be linked to ADHD and other behavioral and emotional problems in the child [9]. Although the precise mechanism of fetal programing is currently unknown, epi- 
genetics suggests that fetal in-utero conditions may reprogram gene function by affecting gene expression without rewriting the genetic code.

If maternal physiological and psychological changes affect the fetus, it is plausible that maternal cognitive activity may also. To date, no studies have explored this theory in humans although animal studies show that maternal exposure to an enriched environment influences cell proliferation in the hippocampus of female mouse fetuses and significantly affects the locomotor activity in female offspring [13]. Environmental enrichment also affects dendritic branching and length, the number of dendritic spines, and the size of synapses of some neuronal populations [14].

\subsection{Objectives}

We aimed to identify and quantify the relation between maternal cognitive (mathematical) activity and changes in fetal nervous system activity by examining fetal brain blood flow (directly related to fetal brain metabolism), and then follow up when the child is approximately three years to assess whether prenatal maternal cognitive activity affects child development, brain potential, and mathematical ability.

\section{Method}

\subsection{Study Design}

Our multi-disciplinary approach combined concepts from the field of mathematical education and medicine. Pregnant women in the 28th to 40th week of pregnancy were given a mathematical questionnaire. Ultrasound Doppler examinations of the fetal brain were performed at various times to measure whether the activity induced changes in fetal brain blood flow resistance and blood flow velocity, both of which possibly reflect fetal brain metabolism. The study was approved by the local Ethics Committee and each participant signed an informed consent.

\subsection{Variables Examined}

The variables examined included fetal sex, ethnicity (Jewish, Arab), maternal age and education, pregnancy duration, and parity.

\subsection{Setting and Equipment}

The study was performed in the obstetric ultrasound unit at the Poriya Medical Center, Israel, using a Doppler ultrasound machine (GE VOLUSONE8 4 - 8 $\mathrm{MHz}$ ). The ultrasound scans were performed by two physicians who specialized in fetal brain scanning and brain blood flow.

\subsection{Mathematical Questionnaire}

The questionnaire developed by the researchers included mathematical literacy, word problems, and geometrical exercises, some developed specifically for this 
study while others were well known, such as the Wechsler Adult Intelligence Scale test [15]. It was designed to be completed within 12 minutes, the level of difficulty of each question could be adjusted according to the mathematical skills of each participant, and also to reduce any possible anxiety. Wechsler and PISA procedures were adhered to.

Due to the demands imposed by the incumbent maternal position during ultrasound examination, the questions were read aloud and the responses were verbal.

Validation of the questionnaire was ensured by first presenting it to a group of 20 women of similar age and education in a neutral environment (to eliminate any anxiety resulting from their presence in the ultrasound room). The activities were documented by videotape. The questionnaire was then adjusted 1) to ensure moderate difficulty (questions that were too easy-all participants answered correctly, or too difficult-all participants answered incorrectly, were excluded); 2) to ensure that ethical principles were adhered to (the test did not discriminate between the backgrounds of individuals); and 3) to ensure that it could be completed within a reasonable time. Once validated, the questionnaire was translated from Hebrew into Arabic.

\subsection{Participants}

Thirty-five pregnant women volunteered to take part in the study. Each participant was rewarded for their involvement. All were at least 20 years old, healthy, and nonsmoking, with uncomplicated, low-risk singleton pregnancy-, and consistent pregnancy dating validated by early first trimester pregnancy testing and ultrasound. Fetal age was 28 - 40 weeks. They were of mixed ethnicity (Arab and Jewish) and heterogeneous with regards to age, parity, education, and fetal sex.

Women who did not feel well during the test or developed contractions were excluded.

\subsection{Data Collection}

Each woman was admitted individually into the ultrasound unit. In order to isolate the influence of the mathematical activity and rule out other influences (for example the effect of anxiety or recumbency), measurements were taken at five points of time: 1) initial measurement; 2) after 10 minutes of recumbent rest (without mathematical activity-to discern whether fetal MCA responses were due to the mathematical activity or recumbency); 3) 8 minutes after the beginning of the mathematical activity; 4 ) at the end of the activity (12 minutes); and 5) 10 minutes after the activity. (See Figure 1 for the experimental protocol.)

\subsection{Measuring Fetal Brain Blood Flow}

From a transverse view, at the level used to measure biparietal diameter, the transducer was moved parallel to this plane towards the base of the skull to identify the Circle of Willis (COW). Color Doppler mapping was used to demonstrate the COW using a $4-8 \mathrm{MHz}$ transabdominal probe (Figure 2(b)). At this 
plane, the MCA is easily demonstrated [16] [17]. To study the fetal brain blood flow, real-time 2D ultrasound Doppler imaging was used to measure PSV and PI in the MCA. After localization of the MCA by color imaging the MCA was sampled with an ultrasound beam as close as possible to an angle of $0^{\circ}$ to the artery closer to the transducer. A sample volume of $2 \mathrm{~mm}$ was placed along the vessel, in the center of the vessel, and approximately $2 \mathrm{~mm}$ distal to the COW (Figure 2).

Within the COW are vessels that communicate between the two internal carotid arteries and the basilar arteries. The main branch of the COW is the MCA, which is the direct continuation of the internal carotid artery and supplies $80 \%$ of the blood flow to the cerebral hemisphere. In its lateral course to the fissure of Sylvius, it supplies the corpus striatum, basal ganglia, insula, and inferior frontal gyrus.

To measure Doppler flow velocity we noted a series of the three highest uniform waves and calculated the average values of PI and PSV (Figure 2(a)). Measurements were repeated three times at each measuring point. All ultra-sonographic measurements were carried out during periods of fetal inactivity and apnea. Throughout measurement, the participant was lying on her back, leaning slightly to the left.

\subsection{Statistical Methods}

The study was designed as a single-group intervention study, with each woman,

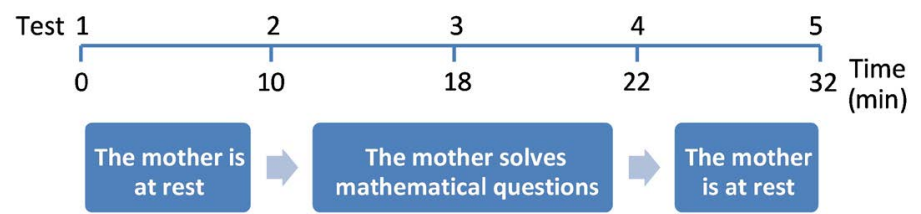

Figure 1. Schematic representation of the experimental protocol. The protocol consists of 5 Doppler tests at the designated time points. During the first and last 10 minutes of the protocol, the mother is at rest, whereas during the 12 minute period in between, the mother solves mathematical questions.

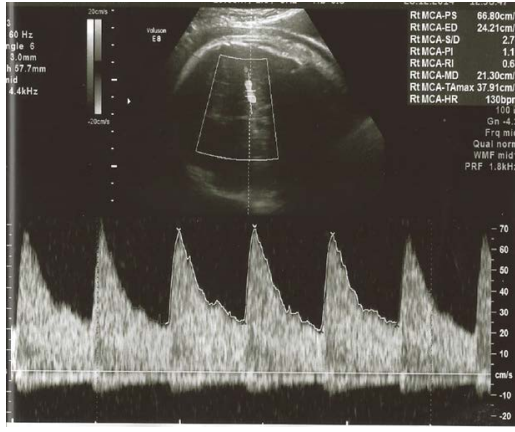

(a)

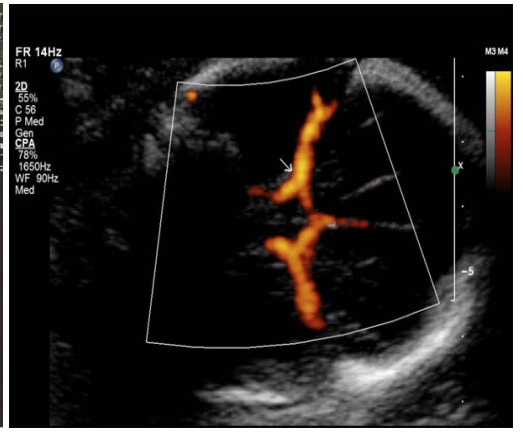

(b)

Figure 2. (a) Doppler measurement of PI/PSV in the MCA; (b) Ultrasound image of the area of measurement of the Doppler in the MCA. PSV = Peak Systolic Velocity, PI = Pulsatility Index, MCA = Middle Cerebral Artery. 
serving as her own control. A two-tailed student's t-test, correlation, and confidence interval were used to analyze the differences between PSV and PI at the different measurements points.

\section{Results}

\subsection{Participant Statistics}

The 35 participants were of mixed population (Arab $n=15$, Jewish $n=20$ ). Ages ranged from 20 to 39 years (average age, 30.14). Gestational age ranged from 28 to 40 weeks (average 31). There were 20 males and 15 female fetuses. The women's parity ranged from 0 - 8 children (average 1.29), and their education ranged from high school graduate to $\mathrm{PhD}$ (average education 14 years).

\subsection{Main Results}

The results suggest that maternal mathematical activity influences fetal brain blood flow, and so may influence fetal brain blood flow rate. We used a two-tail $\mathrm{T}$ test to analyze the differences in PI and PSV between measurements at the different measurement points, shown in Figure 1.

There was a statistically significant difference between PSV values at the end of the mathematical activity (point 4 ) versus PSV values before the onset of mathematical activity (point 1), i.e. a significant decrease in PSV values at the end of the mathematical activity, $\mathrm{p}<0.05$.

There was a statistically significant difference between PI values at the beginning of the test (point 1) versus PI values during the mathematical practice (point 3), i.e., a significant decrease in PI values during mathematical activity, $\mathrm{p}$ $<0.05$. PI values which remained unchanged in response to recumbency (Test 2), reverted back toward baseline levels at Test 4 and remained at, or close, to those levels at Test 5 (Figure 3(a), Figure 3(b)).

Other variables tested: PSV values were higher and PI values were lower with increased gestational age (Figure 4). The differences were statistically significant (2-tailed t-test, $\mathrm{p}<0.001)$.

PSV values were found to be significantly higher $(\mathrm{p}<0.05)$ in male fetuses compared to female fetuses at Test 2 (after recumbency, before mathematical activity) in women with less than 12 years of education.

\section{Discussion and Conclusions}

\subsection{Key Results and Interpretation}

We expected to find a correlation between maternal mathematical activity and the PSV and PI values of fetal MCA, and indeed, our data points to such, as manifested by reduced PI in the MCA, resulting in increased blood flow in the main brain artery. This leads us to hypothesize that maternal mathematical activity during pregnancy might result in both short- and long-term physiological changes in the fetus and, ultimately, in the child. Such results, if corroborated by further studies, are significant and may lead to a revolution in pregnancy man- 
agement protocols aimed to develop mathematical or other cognitive abilities of children (see below).

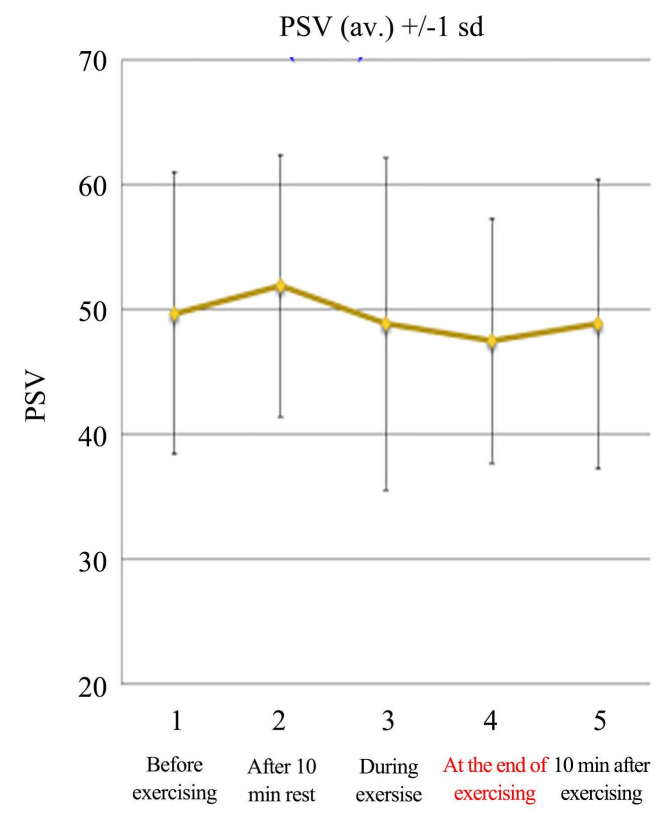

(a)

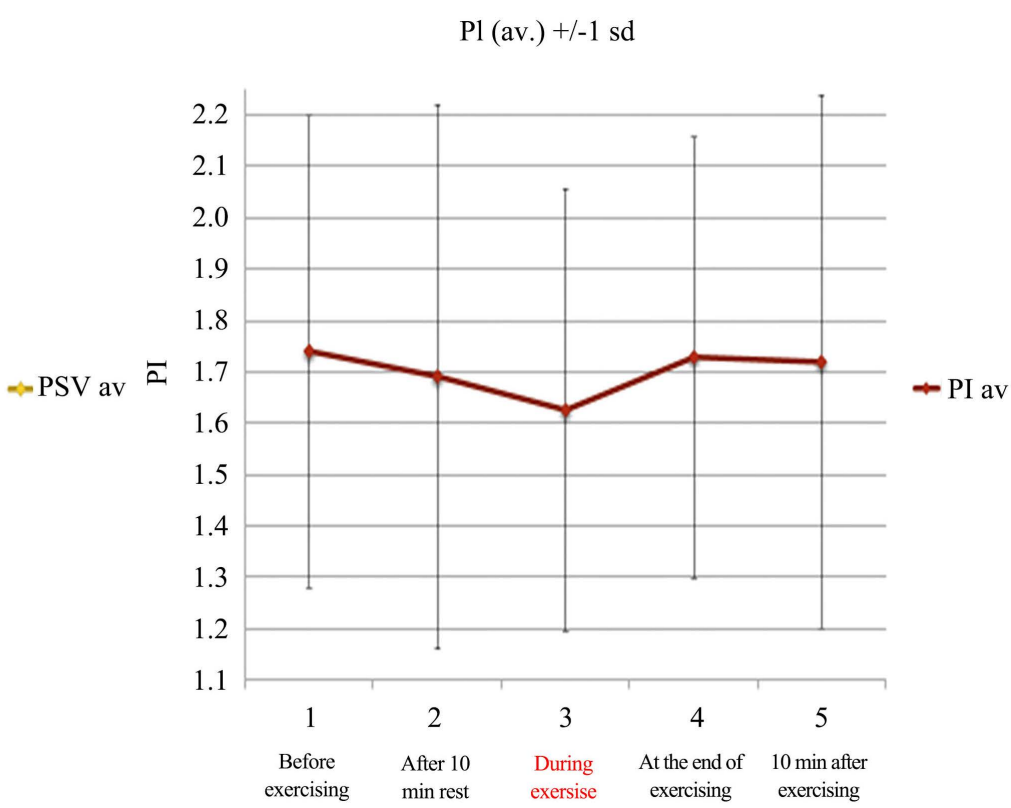

(b)

Figure 3. (a) Peak Systolic Velocity (PSV) values measured at the 5 test points described in Figure 1. Results are presented as means \pm SEM $\left(\mathrm{n}=35,{ }^{\star} \mathrm{p}<0.05\right)$; (b) Pulsatility index (PI) values measured at the five test points described in Figure 1 . Results are presented as means $\pm \operatorname{SEM}\left(\mathrm{n}=35,{ }^{*} \mathrm{p}<0.05\right)$. As noted above, we used a two-tail T test to analyze the differences in PI and PSV between measurements at the different measurement points, shown in Figure 3. There was a statistically significant difference between PSV values at the end of the mathematical activity (point 4) versus PSV values before the onset of mathematical activity (point 1), i.e. a significant decrease in PSV values at the end of the mathematical activity. There was a statistically significant difference between PI values at the beginning of the test (point 1) versus PI values during the mathematical practice (point 3 ), i.e., a significant decrease in PI values during mathematical activity.

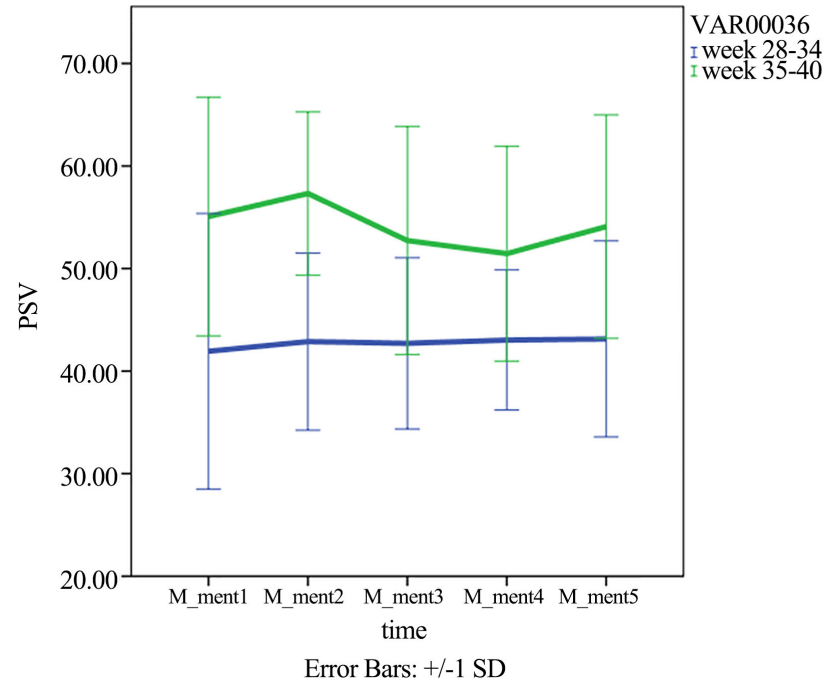

(a)

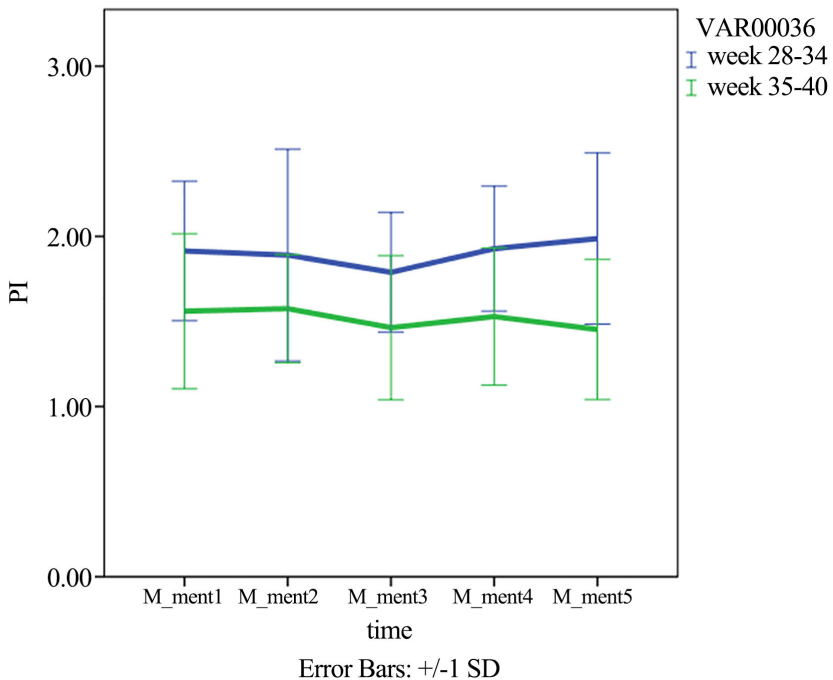

(b)

Figure 4. (a) Peak Systolic Velocity (PSV) values increase with gestational age; (b) Pulsatility Index (PI) values decrease with gestational age. 


\subsection{Further Study}

Our hypothesis is unique in the field: we have not found any study that investigates the effect of maternal cognitive activity on fetal brain functioning. Studies with larger sample sizes can corroborate these results and verify if this can lead to increased mathematical aptitude in the child. In addition, follow-up studies are required to detect any correlation with increased cognitive ability in the child.

To support the findings, and if the Doppler apparatus might not be sensitive enough to demonstrate small differences in blood flow and fetal MCA resistance, other measurable parameters to indicate the fetus's neurobehavior and state of awareness, can be used. Parameters like fetal movements, fetal heart rate variability, and FM/FHR coupling are useful. In addition, maternal stress levels during the activity can be monitored by measuring heart rate, blood pressure, skin conductivity and/or maternal respiration. Blood tests to assess the placental-fetal neuroendocrine axis (especially by placental corticotrophin releasing hormone) may also support the research hypothesis.

Another area worthy of study is whether there is a "window of opportunity" to raise the cognitive potential of fetuses and when it occurs.

Furthermore, one cannot rule out that other types of maternal cognitive activity (apart from mathematics) can affect the fetus and enhance fetal intelligence.

\subsection{Future Child Monitoring for Mathematical Ability}

Once it is shown that maternal mathematical activity enhances fetal neurobehavior and/or fetal brain metabolism, we intend to perform follow-up studies to examine whether enhanced mathematical or other cognitive perception can be observed in the born child at $2-3$ years old. Testing at such a young age is feasible as it has been shown that it is possible to assess children's mathematical abilities already at an early age. Young children do have a perception of mathematics in their everyday life (comparing quantities, creating models, measuring objects, dealing with everyday problems) [18] and babies have a basic concept of quantity long before speech is developed. Preschool children exhibit non-formal mathematical abilities [19] including object counting, spatial orientation, and shape formation [20] [21] [22] sometimes at surprisingly sophisticated levels [23].

\subsection{Future Implications (Long-Term)}

Provided the corroboration of our hypothesis, the possibility of enhanced cognitive abilities in children as a result of maternal mathematical and geometrical activity during pregnancy may revolutionize maternal care and prenatal management, leading to "fetal programing" in which it will be possible to permanently enhance the child's cognitive abilities through maternal mathematical practice during pregnancy. Tools can be designed for the pregnant women to use in order to improve the cognitive abilities of their children. 


\section{Conflicts of Interest}

The authors declare no conflicts of interest regarding the publication of this paper.

\section{References}

[1] DiPietro, J.A., Costigan, K.A., Nelson, P., et al. (2008) Fetal Responses to Induced Maternal Relaxation during Pregnancy. Biological Psychology, 77, 11-19. https://doi.org/10.1016/j.biopsycho.2007.08.008

[2] Copher, D.E. and Huber, C. (1967) Heart Rate Response of the Human Fetus to Induced Maternal Hypoxia. American Journal of Obstetrics \& Gynecology, 98, 320-335. https://doi.org/10.1016/0002-9378(67)90151-2

[3] DiPietro, J.A. (2010) Maternal Influences on the Developing Fetus. Maternal Influences on Fetal Neurodevelopment. Clinical and Research Aspects, 19-32. https://doi.org/10.1007/978-1-60327-921-5_3

[4] DiPietro, J.A., Costigan, K.A. and Gurewitsch, E. (2003) Fetal Response to Induced Maternal Stress. Early Human Development, 74, 125-138. https://doi.org/10.1016/j.earlhumdev.2003.07.001

[5] Monk, C., Myers, M.M., Sloan, R.P., et al. (2003) Effects of Women's Stress Elicited Physiological Activity and Chronic Anxiety on Fetal Heart Rate. Journal of Developmental \& Behavioral Pediatrics, 24, 32-38. https://doi.org/10.1097/00004703-200302000-00008

[6] Monk, C., Sloan, R.P., Myers, M.M., et al. (2004) Fetal Heart Rate Reactivity Differs by Women's Psychiatric Status: An Early Marker for Developmental Risk? Journal of the American Academy of Child \& Adolescent Psychiatry, 43, 283-290. https://doi.org/10.1097/00004583-200403000-00009

[7] DiPietro, J.A., Ghera, M.M. and Costigan, K.A. (2008) Prenatal Origins of Temperamental Reactivity in Infancy. Early Human Development, 84, 569-575. https://doi.org/10.1016/j.earlhumdev.2008.01.004

[8] Wolford, E., Pesonen, A.-K., Lahti, M., et al. (2016) Maternal Depression during Pregnancy Is Associated with Child ADHD Symptoms at 3.5 Years. Psychoneroendocrinology, 71, 33. https://doi.org/10.1016/j.psyneuen.2016.07.090

[9] Uno, H., Eisele, S., Akiko, S., et al. (1994) Neurotoxicity of Glucocorticoids in the Primate Brain. Hormones and Behavior, 28, 336-348. https://doi.org/10.1006/hbeh.1994.1030

[10] Lecaneut, J.P. and Jacquet, A.Y. (2002) Fetal Responsiveness to Maternal Passive Swinging in Low Heart Rate Variability State: Effects of Stimulation Direction and Duration. Developmental Psychobiology, 40, 57-67. https://doi.org/10.1002/dev.10013

[11] Groome, L., Mooney, D., Holland, S., et al. (1999) Behavioral State Affects Heart Rate Response to Low-Intensity Sound in Human Fetuses. Early Human Development, 54, 39-54. https://doi.org/10.1016/S0378-3782(98)00083-8

[12] Querleu, D., Renard, X. and Boutteville, C. (1989) Hearing by the Human Fetus? Seminars in Perinatology, 13, 409-420.

[13] Maruoka, T., Kodomari, I., Yamauchi, R., et al. (2009) Maternal Enrichment Affects Prenatal Hippocampal Proliferation and Open-Field Behaviors in Female Offspring Mice. Neuroscience, 454, 28-32. https://doi.org/10.1016/j.neulet.2009.02.052

[14] Faherty, C.J., Kerley, D. and Smeyne, R.J. (2003) A Golgi-Cox Morphological Anal- 
ysis of Neuronal Changes Induced by Environmental Enrichment. Developmental Brain Research, 141, 55-61. https://doi.org/10.1016/S0165-3806(02)00642-9

[15] Wechsler, D. (2008) Adult Intelligence Scale. 4th Edition, WAIS ${ }^{\oplus}$-IV.

[16] Hershkovitz, R., Erez, O., Sheiner, E., et al. (2003) Elevated Maternal Mid-Trimester Chorionic Gonadotropin Associated with Fetal Cerebral Blood Flow Redistribution. Acta Obstetricia et Gynecologica Scandinavica, 82, 22-27.

[17] Bar, J., Hod, M., Pardo, J., et al. (1997) Effect on Fetal Circulation of Low-Dose Aspirin for Prevention and Treatment of Pre-Eclampsia and Intrauterine Growth Restriction: Doppler Flow Study. Ultrasound in Obstetrics \& Gynecology, 9, 162-265. https://doi.org/10.1046/j.1469-0705.1997.09040262.x

[18] (2002) Early Childhood Mathematics. Promoting Good Beginning. A Joint Position Statement of the National Association for the Education of Young Children (NAEYC) and the National Council of Teachers of Mathematics (NCTM).

[19] Baroody, A.J. (2000) Does Mathematics Instruction for Three-to-Five-Years Old Really Make Sense? Young Children, 55, 61-67.

[20] Douglas, H.C. and Julie, S. (2000) Young Children's Ideas about Geometric Shapes. Teaching Children Mathematics, 6, 482-488.

[21] Hassidov, D. and Ilany, B. (2009) Pre-School Communities and Mathematics Education. Research Issues and Findings. The 33rd Conference of the International Group for the Psychology of Mathematics Education, Thessaloniki, 19-24 July 2009, 64-65.

[22] Hassidov, D. and Ilany, B. (2018) Collaboration between Mathematics Facilitators and Preschool Teachers during the Innovative "Senso-Math" Preschool Program. Mathematics Teacher Education and Development (MTED). Australasia, 20, 154-167.

[23] Clements, D.H. (2001) Mathematics in the Preschool. Teaching Children Mathematics, 7, 270-275. 